\title{
BMC
}

Genetics
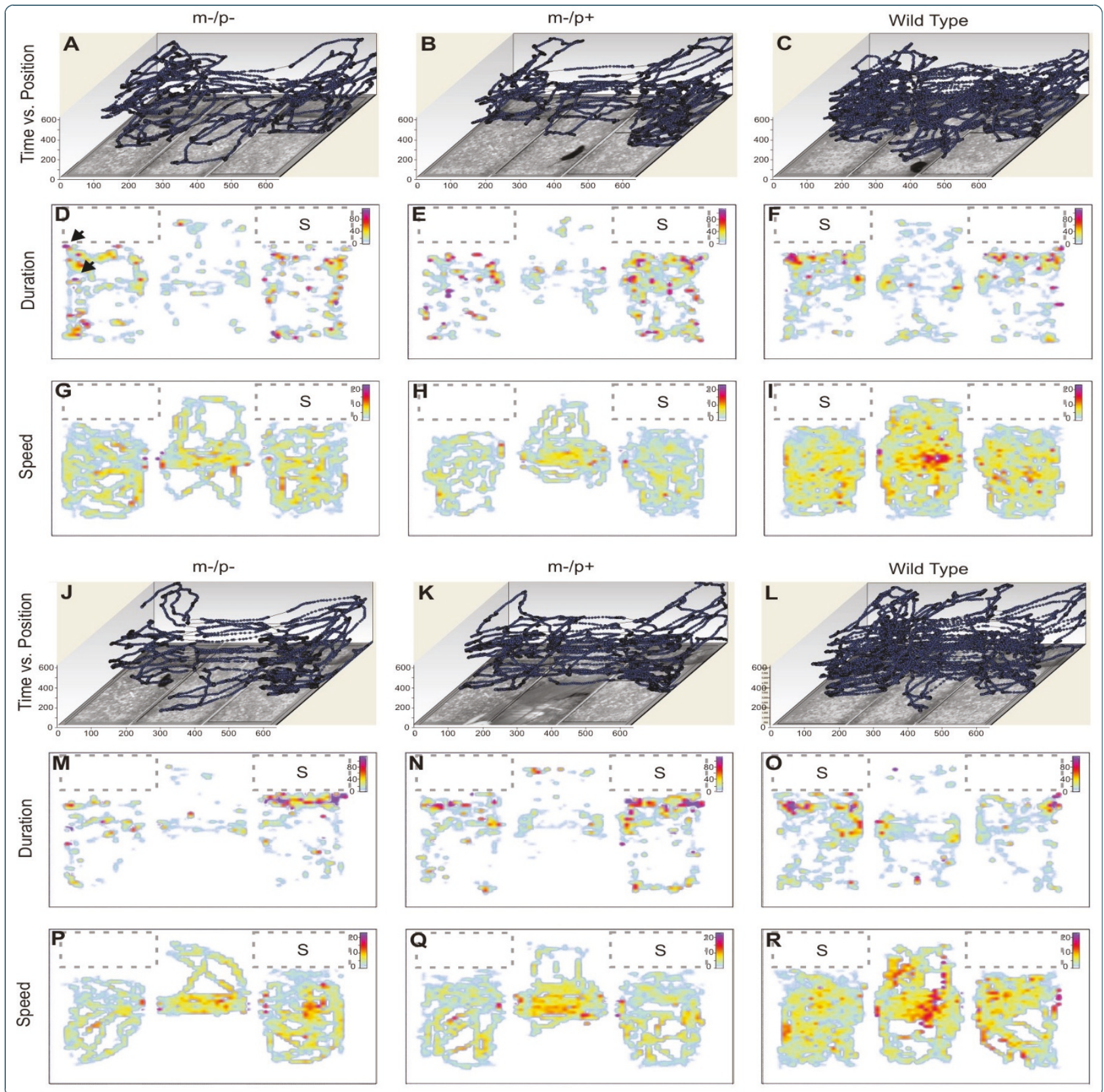

\section{Normal social seeking behavior, hypoactivity and reduced exploratory range in a mouse model of Angelman syndrome}

Allensworth et al. 


\title{
Normal social seeking behavior, hypoactivity and reduced exploratory range in a mouse model of Angelman syndrome
}

\author{
Melody Allensworth', Anand Saha', Lawrence T Reiter ${ }^{1,2,3}$, Detlef H Heck ${ }^{1 *}$
}

\begin{abstract}
Background: Angelman syndrome (AS) is a neurogenetic disorder characterized by severe developmental delay with mental retardation, a generally happy disposition, ataxia and characteristic behaviors such as inappropriate laughter, social-seeking behavior and hyperactivity. The majority of AS cases are due to loss of the maternal copy of the UBE3A gene. Maternal Ube3a deficiency $\left(U b e 3 a^{\mathrm{m}-/ \mathrm{p}+}\right.$ ), as well as complete loss of Ube3a expression $\left(U b e 3 a^{m-/ p-}\right)$, have been reproduced in the mouse model used here.

Results: Here we asked if two characteristic AS phenotypes - social-seeking behavior and hyperactivity - are reproduced in the Ube3a deficient mouse model of AS. We quantified social-seeking behavior as time spent in close proximity to a stranger mouse and activity as total time spent moving during exploration, movement speed and total length of the exploratory path. Mice of all three genotypes (Ube $3 a^{\mathrm{m}+/ \mathrm{p}+}$, Ube $3 a^{\mathrm{m}-1 \mathrm{p}+}, \mathrm{Ube} 3 \mathrm{a}^{\mathrm{m}-\mathrm{p}^{\mathrm{p}}}$ ) were tested and found to spend the same amount of time in close proximity to the stranger, indicating that Ube3a deficiency in mice does not result in increased social seeking behavior or social dis-inhibition. Also, Ube3a deficient mice were hypoactive compared to their wild-type littermates as shown by significantly lower levels of activity, slower movement velocities, shorter exploratory paths and a reduced exploratory range.

Conclusions: Although hyperactivity and social-seeking behavior are characteristic phenotypes of Angelman Syndrome in humans, the Ubeza deficient mouse model does not reproduce these phenotypes in comparison to their wild-type littermates. These phenotypic differences may be explained by differences in the size of the genetic defect as $\sim 70 \%$ of AS patients have a deletion that includes several other genes surrounding the UBE3A locus.
\end{abstract}

\section{Background}

Angelman syndrome (AS) is a neurogenetic disorder characterized by severe developmental delay with mental retardation, language delays, increased susceptibility to seizures and unique behavioral characteristics such as inappropriate laughter, social-seeking behavior and hyperactivity $[1,2]$. The prevalence of AS in the general population is between $1: 10,000$ and 1:40,000 [3]. Most cases of AS are caused by loss of function of the maternal copy of the $U B E 3 A$ gene, although paternal uniparental disomy, imprinting center mutations and maternally inherited loss of function mutations in $U B E 3 A$ can occur as well $[4,5]$. The $U B E 3 A$ gene

\footnotetext{
* Correspondence: dheck@uthsc.edu

'Department of Anatomy and Neurobiology, University of Tennessee Health Science Center, Memphis, TN 38139, USA

Full list of author information is available at the end of the article
}

encodes an E3 ubiquitin protein ligase, which is involved in targeting proteins for mono-ubiquitination and subsequent cellular processes such as degradation or trafficking to other parts of the cell. In the brain $U B E 3 A$ exhibits preferential maternal allele expression with highest levels of expression in the cerebellum and hippocampus [6]. Behavioral abnormalities of AS include inappropriate laughter, a generally happy disposition, impaired verbal skills, social dis-inhibition or social seeking behavior and hyperactivity [1-3]. Social seeking behavior is expressed as a dis-inhibited and unselective desire of AS individuals to seek attention from both caregivers and strangers.

Some studies have reported overlapping features between autism and AS especially when the maternally inherited deletions include not only the UBE3A gene, but also genes like CYFIP1, a gene coding for a protein that

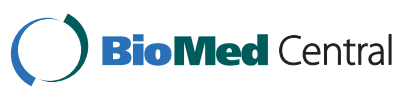


directly interacts with the Fragile $\mathrm{X}$ protein FMRP, that are proximal to the $U B E 3 A$ locus $[7,8]$. However, social seeking behavior is uncharacteristic of autism and AS patients show a strong interest in social interaction from early infancy [9]. With their verbal communication impaired, these individuals typically communicate through facial expressions, gestures and body postures [7,10].

Jiang and colleagues were the first group to engineer a mouse model of AS via knock out of exon 2 of the mouse Ube $3 a$ gene [11]. The Ube $3 a$ deficient mouse model is often studied in the context of molecular studies that are aimed at finding the many roles of the $U B E 3 A$ gene [6]. Only a few behavioral studies have been performed with Ube $3 a$ deficient mice [11-13] and none have addressed the social behaviors in the mouse model as compared to AS individuals. Moy et al. used a social interaction task similar to the one employed here to study social behavior in five mouse lines with genetic defects known to be related to Autism Spectrum Disorders in humans [14]. The behavioral abnormalities in these mice included a reduced social interest suggesting that the genes involved affected social behavior in both humans and mice.

The purpose of this study was to investigate social interest in a stranger mouse and exploratory activity in a mouse model of Angelman syndrome. We hypothesized that the human hyperactivity and social seeking phenotypes found in AS patients would be reproduced in Ube3a deficient mice.

\section{Results}

All tests were performed with adult male mice $8-12$ weeks of age that were either homozygous for the Ube3a null allele (Ube3a $a^{m-/ p-}, n=11$ ) or inherited the null allele through the maternal germline $\left(U b e 3 a^{m-/ p+}\right.$, $n=25$ except for measurements of area covered where $n=23)$. Wild-type littermates (Ube3 $\left.a^{m+/ p+}, n=36\right)$ of these animals were used as controls. The construction and breeding of the Ube3a deficient mice have been described by Jiang and colleagues [11].

The results section is divided into two segments: first, we report results from analyzing the entire length of the path, covering all of the arena (gray area in Figure 1A) under control conditions (with the two small cages empty) and under test conditions (with a stranger mouse present in one of the cages). Second, we describe results from analyzing the behavior directly in front of the two small cages, differentiating between the empty cage and the cage with the stranger mouse. Here, pathway analysis was limited to within the two small zones shown in gray in Figure 1B.

\section{Whole Arena Measurements}

Representative examples of exploratory behavior of Ube $3 a$ deficient and wild-type mice under control and

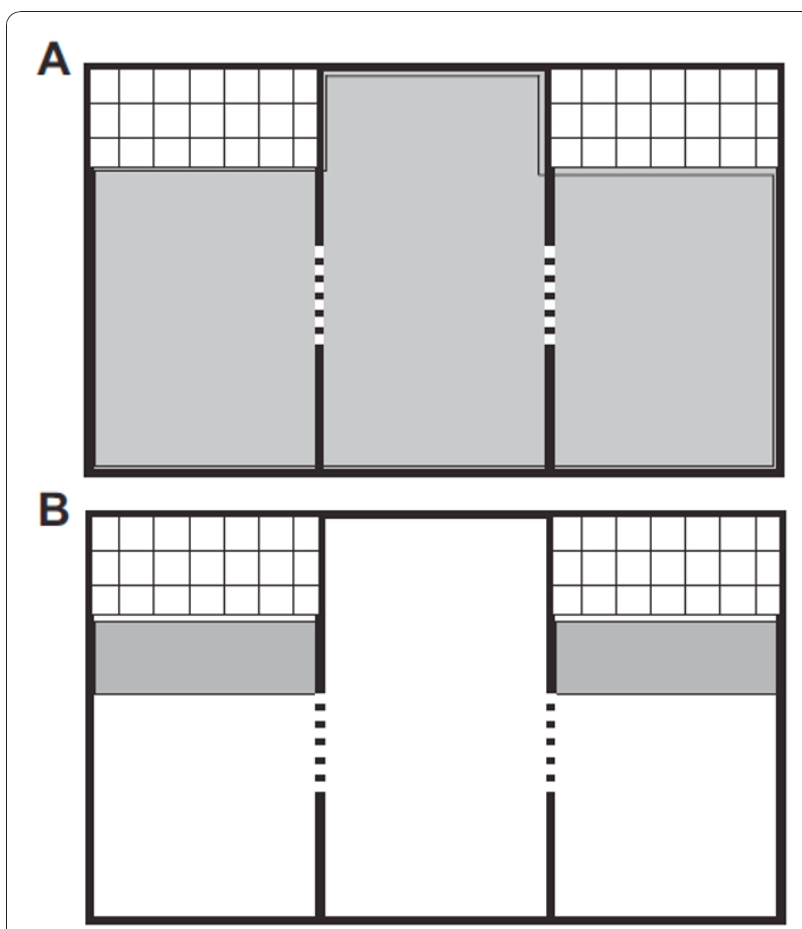

Figure 1 Schematic drawing showing the outline of the arena with its three chambers and the two small cages and the areas used for movement path analysis. The dividing walls had doors in the center (indicated by dashed lines) through which the mice could freely move between chambers. (A) The area shown in gray is the area within which the mouse's movements were tracked for the analysis of behavior across the whole arena. (B) For the analysis of social interest in the stranger mouse the analysis of the movement paths was limited to the two gray rectangular areas in front of the two cages.

test conditions (without and with a stranger mouse present, respectively) are visualized for qualitative comparison in Figure 2 as movement path vs. time plots (Figure $2 \mathrm{~A}-\mathrm{C}, \mathrm{J}-\mathrm{L}$ ), and color maps for duration (Figure 2D-F, $\mathrm{M}-\mathrm{O}$ ) and movement speed (Figure 2G-I, P-R). Quantitative comparisons are shown in subsequent figures.

Movement path vs. time plots show the exploratory paths overlying the video image of the arena with time plotted along the $\mathrm{z}$-axis (Figure $2 \mathrm{~A}-\mathrm{C}, \mathrm{J}-\mathrm{L}$ ). Visual comparison of the path vs. time plots shows that both Ube $3 a$ deficient mice had shorter exploratory paths and covered less area in their exploration than wild-type mice under both control (Figure 2A-C) and test conditions (Figure 2J-L). The quantitative comparison of path length under control and test conditions revealed that Ube $3 a^{\mathrm{m}-/ \mathrm{p}-}$ and $U b e 3 a^{\mathrm{m}-/ \mathrm{p}+}$ mice had significantly shorter exploratory paths than their wild-type litter mates, under both control and test conditions (Figure $3 \mathrm{~A})(F[5,138]=33.29, p \leq 0.001$, Tukey post hoc $(\mathrm{TPH})$ results for pairwise comparison to wild type litter mates under control conditions: Ube $3 a^{\mathrm{m}-/ \mathrm{p}-}, p \leq 0.001$, 


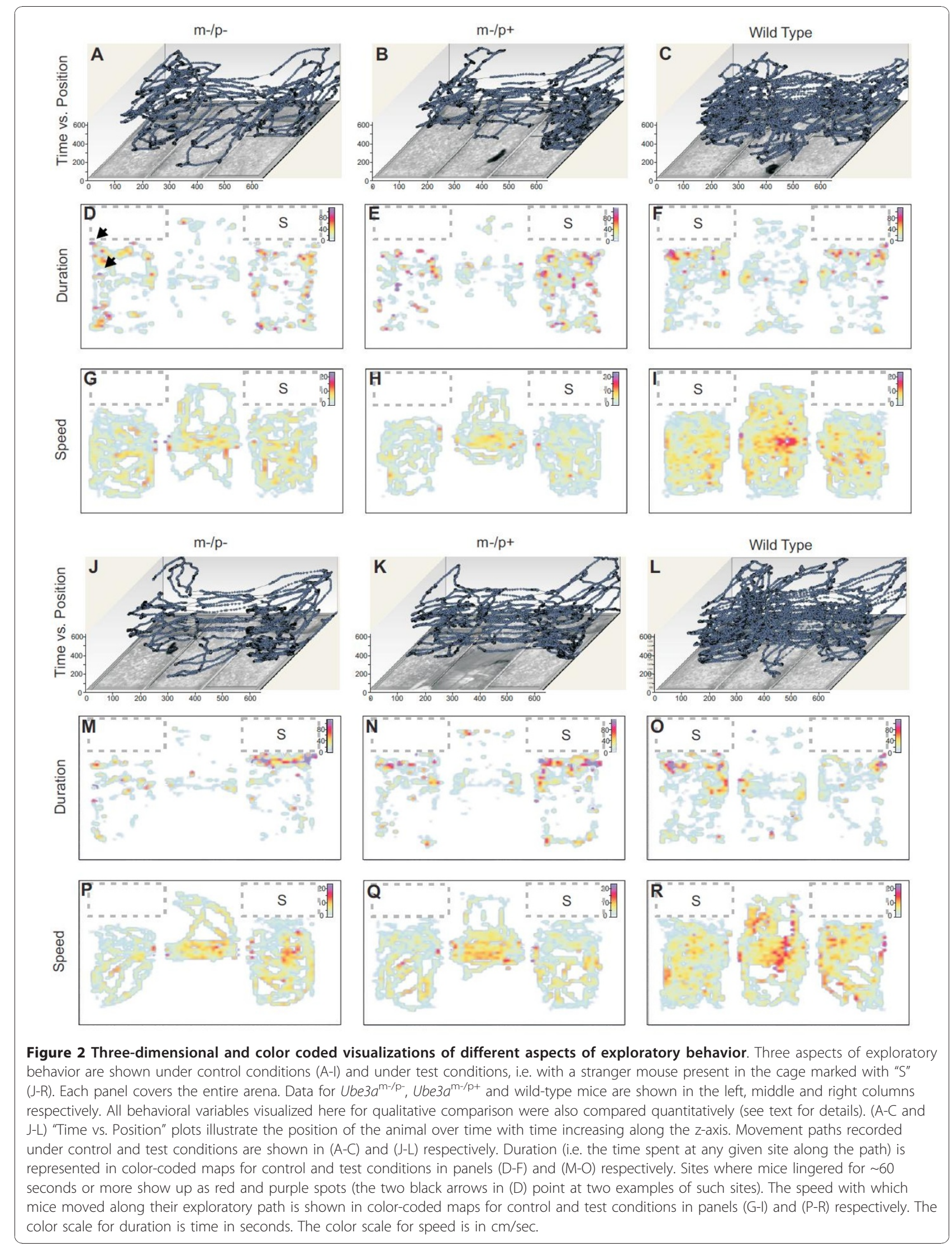


on the left and from test trials (with stranger present) on the right. Bar colors correspond to genotypes (Ube $3 a^{m-/ p^{-}}=$white bar, Ube $3 a^{\mathrm{m}-/ \mathrm{p}+}=$ light gray, wild-type = dark gray). (A) The total length of the exploratory path was significantly shorter for Ube3a deficient compared to wild-type mice. (B) The area covered by the exploratory path was significantly smaller for Ube3a deficient compared to wild-type mice. For all three genotypes the area covered was larger during the control trial (left) than during the test trial. (C) Ube3a deficient mice spent significantly less time moving than wild-type mice during both control and test conditions. (D) The average movement speed was significantly lower for Ube3a deficient compared to wild-type mice. Symbols: * indicates a significant difference between the Ube3a deficient and the wildtype mice. $\neq$ indicates a significant difference between control and test conditions within the same group of mice. Error bars are SEM.
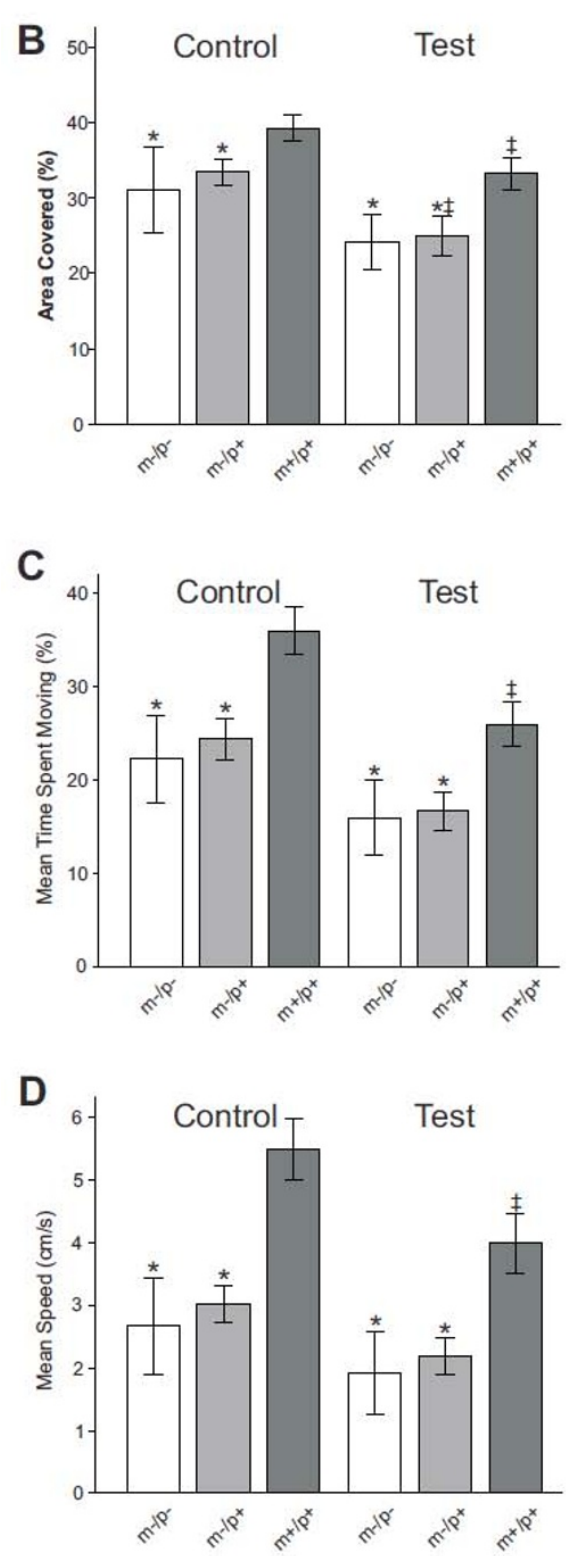

Figure 3 Various measures of exploratory activity are significantly lower in Ube3a deficient mice compared to wildtype littermates. Each bar graph shows results from control trials mdiff $=-1690.23$, serr $=242.46$ and $U$ Ue $3 a^{\mathrm{m}-/ \mathrm{p}+}, p \leq$ 0.001 , mdiff $=-1455.57$, serr $=183.22$; TPH results for pairwise comparison to wild type litter mates under test conditions: Ube $3 a^{\mathrm{m}-/ \mathrm{p}-}, p \leq 0.001$, mdiff $=-1223.17$, serr = 242.46; Ube3a $a^{\mathrm{m}-/ \mathrm{p}+}, p \leq 0.001$, mdiff $=-1061.58$, serr $=$ 183.22). Similarly, quantitative comparison of the area covered by the paths (Figure 3B) showed that wild-type mice covered a significantly larger proportion of the arena than Ube3a deficient mice $(F[5,134]=20.89, p \leq$ $0.005, \mathrm{TPH}$. results for pairwise comparison to wild type litter mates under control conditions: Ube $3 a^{\mathrm{m}-/ \mathrm{p}-}, p \leq$ 0.002, mdiff $=-8.16$, serr $=2.07 ;$ Ube $3 a^{\mathrm{m}-/ \mathrm{p}+}, p \leq 0.005$, mdiff $=-5.81$, serr $=1.6$; TPH results for pairwise comparison to wild type litter mates under test conditions: Ube $3 a^{\mathrm{m}-/ \mathrm{p}+}, p \leq 0.001$, mdiff $=-8.22$, serr $=1.6$; Ube $3 a^{\mathrm{m}-/ \mathrm{p}-}, p \leq 0.001$, mdiff $=-9.01$, serr $\left.=2.07\right)$. There were no significant differences in path length or area covered between $U b e 3 a^{\mathrm{m}-/ \mathrm{p}^{-}}$and $U b e 3 a^{\mathrm{m}-/ \mathrm{p}^{+}}$mice under either condition.

Color maps for duration of stay (Figure 2D-F, M-O) show sites were mice lingered for more than 60 seconds as red and purple spots (two examples marked by arrows in Figure 2D). Both $U b e 3 a^{\mathrm{m}-/ \mathrm{p}-}$ and $U b e 3 a^{\mathrm{m}-/ \mathrm{p}+}$ mice (Figure 2D-F) lingered frequently for 60 seconds or more at various sites distributed across most of the two side chambers with a preference of lingering close to a wall (Figure 2D,E). Mice rarely lingered in the middle chamber.

By comparison, the wild-type mouse had overall fewer places where it lingered for more than 60 seconds and lingering sites were concentrated in front of the two empty cages (Figure 2F). A quantitative comparison of lingering behavior as the proportion of time the mice spent moving during the $10 \mathrm{~min}$ trials revealed that wild-type mice spent significantly more time moving than did Ube3a deficient mice $(F[5,138]=30.99$; $p \leq 0.001$; TPH results for pairwise comparison to wild type litter mates under control conditions: Ube $3 a^{\mathrm{m}-/ \mathrm{p}-}$, 
$p \leq 0.001$, mdiff $=-13.77$ serr $=2.33 ; U$ Ube $3 a^{\mathrm{m}-/ \mathrm{p}+}, p \leq$ 0.001 , diff $=-11.62$, serr $=1.76$, TPH results for pairwise comparison to wild type litter mates under test conditions: Ube $3 a^{\mathrm{m}-/ \mathrm{p}-}, p \leq 0.001$, mdiff $=-10.02$, serr $=$ 2.33; Ube $3 a^{\mathrm{m}-/ \mathrm{p}+}, p \leq 0.001$, mdiff $=-9.33$, serr $\left.=1.76\right)$. Comparison of the color maps for speed (Figure $2 \mathrm{G}-\mathrm{I}$, P-R) show that Ube3a deficient mice generally moved at a slower speed than their wild-type littermates. Quantitative comparison revealed significantly lower movement speeds in both Ube3a deficient genotypes compared to wild-type littermates $(F[5,138]=32.52, p \leq 0.001$; TPH results for pairwise comparison to wild type litter mates under control conditions: Ube $3 a^{\mathrm{m}-/ \mathrm{p}-}, p \leq 0.001$, mdiff $=$ -2.83, serr $=0.41 ;$ Ube $3 a^{\mathrm{m}-/ \mathrm{p}+}, p \leq 0.001$, diff $=-2.47$, serr $=0.31$; TPH results for pairwise comparison to wild type litter mates under control conditions: $U b e 3 a^{\mathrm{m}-/ \mathrm{p}-}$, $p \leq 0.001$, mdiff $=-2.07 ;$ serr $=0.41 ;$ Ube $a^{\mathrm{m}-/ \mathrm{p}+}, p \leq$ 0.001 , mdiff $=-1.81$, serr $=0.31)$ (Figure 3D). This difference was independent of social context, i.e. of the presence or absence of a stranger mouse. There was no significant difference in movement speed between $U b e 3 a^{\mathrm{m}-/ \mathrm{p}-}$ and $U b e 3 a^{\mathrm{m}-/ \mathrm{p}+}$ mice under either condition.

During the test trials (with a stranger mouse present) mice from all three genotypes spent extended amounts of time in front of the cage with the stranger mouse (cage marked "S" in Figure 2M-R) signified by the accumulation of lingering sites (red/purple dots). This was interpreted as social interest in the stranger mouse and was quantified as the total amount of time the mice spent inside the area in front of the cage. We also measured the number of visits or entries into the area in front of the stranger's cage. A detailed description of results for the behaviors directly in front of cages is given below.

We consistently observed that mice of all three genotypes moved slower, had shorter track lengths and spent less time moving during the second trial, i.e. when the stranger mouse was present in one of the cages. The decreases in these variables were comparable in magnitude between genotypes but significant differences for each variable were only seen in the wild-type mice (Figure 3) (TPH results for pairwise comparison between trials: Track length: $p \leq 0.001$, mdiff $=-907.9$, serr $=$ 165.88; Velocity: $p \leq 0.001$, mdiff $=-1.51$, serr $=0.28$; Activity: $p \leq 0.001$, mdiff $=-10.03$, serr $=1.59)$. We wanted to know whether this decrease in exploratory activity during the test trial was related to the presence of the stranger mouse or due to reduced drive to explore a now familiar arena. Control experiments were conducted in which the mice explored the arena twice without a stranger mouse present. All other procedures were kept identical to the social tests. These control experiments were conducted only with $U b e 3 a^{\mathrm{m}-/ \mathrm{p}+}(n=$ $5)$ and wild-type $(n=5)$ mice, as we had not found any differences between $U b e 3 a^{\mathrm{m}-/ \mathrm{p}-}$ and $U b e 3 a^{\mathrm{m}-/ \mathrm{p}+}$ in exploratory activity. During the second trial of the control experiments movement speed, track length and time spent moving decreased for both the $U b e 3 a^{\mathrm{m}-/ \mathrm{p}+}$ and wild-type mice. The observed decreases were comparable in magnitude to those observed in the social experiments. Decreases in time spent moving reached significance for both $U b e 3 a^{\mathrm{m}-/ \mathrm{p}+}$ and wild-type mice $(F[3,16]=27.92, p \leq 0.001$; TPH results for pairwise comparison between trials: Ube $3 a^{\mathrm{m}-/ \mathrm{p}+}, p \leq 0.001$, mdiff $=-11.1$, ser $=2.22$, Ube $3 \mathrm{a}^{\mathrm{m}+/ \mathrm{p}+}, p \leq 0.001$, diff $=$ -11.98 , serr $=2.22$ ). Thus, the observed decreases in exploratory activity in the second trial of the social test were not dependent on the presence of a stranger mouse.

\section{Behavior in front of cages}

Social interactions with the stranger mouse were quantified as the amount of time the test mice spent in a small area directly in front of the stranger's cage (shaded areas in Figure 1B). Comparison of the total time mice spent directly in front of either cage (duration) revealed that mice of all genotypes spent significantly more time in front of the stranger's cage than in front of the empty cage (Figure 4A) $(F[11,272]=18.62, p \leq 0.001$; TPH results for pairwise comparison between cages during presence of a stranger mouse (test conditions): $U b e 3 a^{\mathrm{m}-/ \mathrm{p}-}, p \leq 0.001$, mdiff $=137.7$, serr $=28.8 ;$ Ube $3 a^{\mathrm{m}-/ \mathrm{p}+}, p \leq 0.001$, mdiff $=$ 136.67, serr $=19.5$; Ube3a $\mathrm{a}^{\mathrm{m}+/ \mathrm{p}^{+}}, p \leq 0.001$, mdiff $=87.54$, serr $=15.92)$. There were no significant differences between wild type and Ube3a deficient mice for the amount of time spent in front of the stranger's cage. Under control conditions, mice of all genotypes spent the same amount of time in front of both cages, showing that there was no preexisting preference for either cage.

Analysis of the number of visits, i.e. the number of times mice walked into the areas in front of the cages, revealed significant differences between groups (Figure $4 \mathrm{~B})$. Under control conditions both $U b e 3 a^{\mathrm{m}-/ \mathrm{p}-}$ and Ube $3 a^{\mathrm{m}-/ \mathrm{p}+}$, had significantly fewer visits to either of the two cages than wild-type mice $(F[11,272]=13.96, p \leq$ 0.001 ; TPH results for pairwise comparison with wildtype litter mates for the cage that was empty during control and test trials: Ube $3 a^{\mathrm{m}-/ \mathrm{p}-}, p \leq 0.015$, mdiff $=$ -8.57, serr $=2.33 ;$ Ube $3 a^{\mathrm{m}-/ \mathrm{p}+}, p \leq 0.004$, mdiff $=-7.15$, serr $=1.79$. TPH results for pairwise comparison with wild-type litter mates for the cage that was empty during control but housed the stranger mouse during test trials: Ube $3 a^{\mathrm{m}-/ \mathrm{p}-}, p \leq 0.001$, mdiff $=-11.94$, serr $=2.33$; Ube $3 a^{\mathrm{m}-/ \mathrm{p}+}, p \leq 0.015$, mdiff $=-8.72$, serr $\left.=1.79\right)$. Also with the stranger present, Ube3a deficient mice still visited the stranger's cage significantly less often than the wildtype mice $(F[11,272]=13.96$; TPH results for pairwise comparison with wild-type litter mates: Ube $3 a^{\mathrm{m}-/ \mathrm{p}-}, p \leq$ 0.041 mdiff $=-7.84$, serr $=2.33$, Ube $3 a^{\mathrm{m}-/ \mathrm{p}+}, p \leq 0.001$, mdiff $=-8.72$, serr $=1.79$ ). 

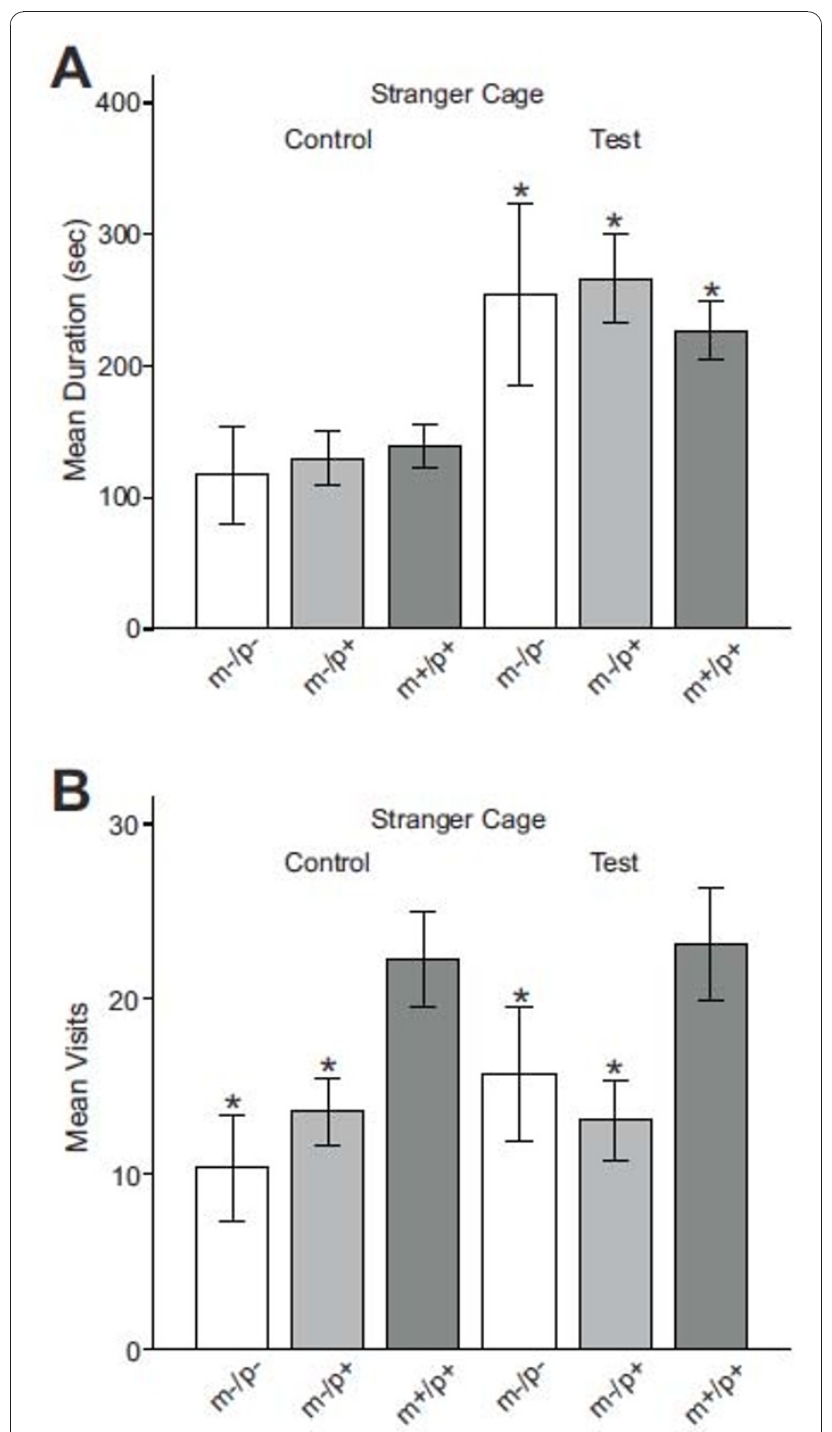

Figure 4 Analysis of behavior in the area directly in front of the cages with and without the stranger mouse. Each bar graph shows results from control trials on the left and from test trials (with stranger present) on the right. Bar colors correspond to genotypes (Ube $3 a^{\mathrm{m}-/ \mathrm{p}-}=$ white bar, Ube $3 a^{\mathrm{m}-/ \mathrm{p}+}=$ light gray, wild-type $=$ dark gray). (A) Time spent in front of the cage when the stranger was present (test trial) compared to the same cage when it was empty (control trial). Asterisks indicate significant differences between control and test conditions. (B) Mean number of visits to the stranger's cage during control and test conditions. Asterisks indicate a significant difference between the Ube3a deficient and the wildtype mice. Error bars are SEM.

The analysis of the paths within the areas in front of the cages revealed similar differences between $U b e 3 a$ deficient and wild type mice in movement speed, exploratory path lengths and time spent moving found for the entire path. Ube3a deficient mice spent significantly less time moving in the areas in front of the cages than their wild-type litter mates under control and test conditions $(F[11,272]=20.89, p \leq 0.001$; TPH results for pairwise comparison with wild-type litter mates during control conditions: $U b e 3 a^{\mathrm{m}-/ \mathrm{p}-}, p \leq 0.001$, mdiff $=-10.72$, serr $=2.02 ;$ Ube $3 a^{\mathrm{m}-/ \mathrm{p}+}, p \leq 0.001$, mdiff $=-9.06$, serr $=1.54$. TPH results for pairwise comparison with wild-type littermates during test conditions: Ube $3 a^{\mathrm{m}-/ \mathrm{p}-}, p \leq 0.001$, mdiff $=-8.73$, serr $=2.02$; Ube $3 a^{\mathrm{m}-/ \mathrm{p}+}, p \leq 0.001$, mdiff $=-7.96$, serr $\left.=1.54\right)$. There was no significant difference between $U b e 3 a^{\mathrm{m}-/ \mathrm{p}-}$ and Ube $3 a^{\mathrm{m}-/ \mathrm{p}+}$ mice in any of the variables. As before, these differences were in not affected by the presence of the stranger mouse.

We also analyzed freezing behavior in the whole field and in front of the cages. There was no significant difference in the number of freezing events between any of the genotypes or between experimental conditions.

\section{Discussion}

We tested mice with either maternal $\left(U b e 3 a^{\mathrm{m}-/ \mathrm{p}+}\right)$ or complete $\left(U b e 3 a^{\mathrm{m}-/ \mathrm{p}-}\right)$ loss of $U b e 3 a$ expression for their social interest in a stranger mouse as well as for their levels of activity and patterns of exploratory behavior. The main findings of our study are that social seeking behavior and hyperactivity, both of which are characteristic behavioral phenotypes of human Angelman syndrome patients $[1,2,15,16]$, were not observed in either form of $U b e 3 a$ deficient mice. Ube3a deficient mice showed the same interest in spending time with a stranger mouse as did their wild-type littermates, indicating normal social behavior. The exploratory behavior of Ube3a deficient mice revealed reduced activity compared to their wild-type littermates. Thus, in contrast to Angelman syndrome, were hyperactivity is consistently described as a behavioral phenotype, complete or maternal loss of Ube3a expression in mice results in hypoactivity. Loss of Ube3a expression also causes mild cerebellar ataxia in both humans and mice $[1,2,10,13]$. Considering possible effects of ataxia on the measurement of activity it is important to note that the activity was determined as the amount of time the mice spent moving faster than a very low minimum speed $(0.10$ $\mathrm{cm} / \mathrm{sec}$ ). Analysis of exploratory paths showed that Ube3a deficient mice were clearly able to move much faster than the threshold-speed for activity registration. Thus, ataxia is unlikely to be the cause of hypoactivity in mice.

Interestingly, the numbers of visits to the areas in front of the cage with the stranger mouse were not different in any genotype for control (no mouse present) and test conditions (stranger mouse present). This suggests that the presence of the stranger mouse did not cause any of the mice to change their exploratory path to increase the number of encounters, but instead that visits to the cages were rather chance events during 
exploration. The extended stay in front of the stranger's cage, however, was clearly related to the presence of the stranger. We did see, however, that Ube3a deficient mice visited the cages significantly less often than did wild type mice. We suggest that this difference is not related to social context but rather due to the difference in overall exploratory activity. The difference in the number of visits to the areas in front of the cages amounted to $37.7 \%$ fewer visits of Ube3a deficient compared to wild-type mice. This reduction is of similar magnitude as the $37.3 \%$ difference in the amount of time spent moving (with Ube3a deficient mice moving less).

All mice showed a general reduction in various measures of activity (speed, track length, time spent moving) during the second or test trial. However, those reductions in activity were also observed in control experiments where no stranger mouse was present during the second trial. Thus, the reduced activity was independent of the stranger's presence and likely the result of reduced drive to explore the then familiar arena during the second trial.

In order to determine the value of the Ube3a deficient mouse as a model for Angelman syndrome it will be important to understand whether the phenotypic differences between patients and Ube3a deficient mice reported here are based on species specific effects of loss of Ube3a expression, on differences in the extent of the genetic defects or on a combination of both. It is important to point out, that the majority of Angelman patients have contiguous gene deletions, i.e. have lost expression of more than one gene. There are several molecular classes of Angelman syndrome including 15q11-q13 deletion, uniparental disomy, imprinting defects and $U B E 3 A$ gene mutation. The most common of these is a deletion of maternal origin on chromosome $15 \mathrm{q} 11-\mathrm{q} 13$, which accounts for about $70 \%$ of AS cases [17]. Patients with the deletion phenotype tend to have a more severe phenotype, for example severe microcephaly, more severely impaired communication and more severe seizures than patients with other molecular classes of $U B E 3 A$ deficiency [17]. These deletions can be further categorized into class I and class II deletions. Patients with the larger class I deletions that encompass the locus for the FRMP interacting protein CYFIP1 appear to have an increased risk for autism as compared to patients with class II deletions [8]. It should also be noted that individuals with Prader-Willi Syndrome (PWS) as a result of maternal uniparental disomy (i.e. two maternal copies of 15 and thus no paternal specific expression at the SNRPN locus) have also been reported as having more autism like features than PWS deletion individuals [18]. These phenotypic differences among deletion, mutation and even uniparental disomy individuals may help to explain some of the conflicting literature in regards to social behavior observed in Angelman syndrome. Similarly, the fact the Ube3a deficient mice lack expression of only one gene might be at least partially responsible for the phenotypic differences between Angelman patients and $U b e 3 a$ deficient mice reported here. A recently published study used a new mouse model with a large maternal deletion from Ube $3 a$ to Gabrb3. This mouse model reflects the contiguous human gene deletions more closely and a comparative study between both genetic models would be informative about the role of specific role of Ube3a in hyperactivity, social and other behaviors.

All Angelman syndrome patients have significant developmental delay in cognitive and motor functions $[3,19]$. Weather the behavioral patterns observed here in adult mice changes with age and weather those changes parallel development in humans is thus another important question to address in follow-up studies.

\section{Conclusions}

Although the mouse model of Ube3a deficiency used here shares physiological and phenotypic features with Angelman patients, we show that social seeking behavior and hyperactivity are not reproduced. The majority of AS patients have a maternally derived deletion, which encompasses several genes including $U B E 3 A$. Individuals with maternally inherited mutations affecting only $U B E 3 A$ tend to have a milder phenotype than the individuals with deletions [6]. The mice tested here are more similar to the individuals with point mutations since they are lacking only the Ube3a protein and do not have a contiguous gene deletion [11]. It remains to be shown whether the phenotypical differences between Ube $3 a$ deficient mice and Angelman patients are based on species-specific differences, differences in genetic conditions or a combination of both.

\section{Methods}

The construction and breeding of the $U b e 3 a$ deficient mice used for these studies have been previously described [11] and these animals have previously been used for behavioral studies by our group [13]. The mice used for this study were $98 \%$ congenic to C57BL/6J according to SNP genotyping performed at Jackson Laboratory (Bar Harbor, Maine). All mice used in this study were raised and all experiments performed in accordance with procedural guidelines approved by the University of Tennessee Health Science Center Animal Care and Use Committee. Principles of laboratory animal care (NIH publication No. 86-23, rev. 1996) were followed.

All behavioral tests were performed with adult male mice 8 - 12 weeks of age that were either homozygous 
for the Ube3a null allele (Ube3a $\left.a^{m-/ \mathrm{p}-}, n=11\right)$ or inherited the null allele through the maternal germline $\left(U b e 3 a^{m-/ p+}\right.$, $n=25$, except for measurements of area covered where $n$ $=23$ ). Wild-type littermates (Ube $3 \mathrm{a}^{\mathrm{m}+/ \mathrm{p}+}, n=36$ ) of these animals were used as controls. Mice were housed in standard cages with ad libitum food and water access with 12hour light/dark cycles. Mice used as strangers in the social test were wild-type C57BL/6 mice that were unrelated to the test animals and had not been previously in contact with test animals.

\section{Social Behavior Test}

The social behavior test was designed based on the test developed by Moy et al. [14,20] to quantitatively evaluate the interaction of the test mice with a stranger mouse and at the same time evaluate exploratory behavior. Tests were performed in a rectangular Plexiglas arena $(63 \mathrm{~cm} \mathrm{~L} \times 42 \mathrm{~cm} \mathrm{~W} \times 22 \mathrm{~cm} \mathrm{H})$, which was subdivided in to three rectangular chambers of equal size $(21 \mathrm{~cm} \mathrm{~L} \times 42 \mathrm{~cm} \mathrm{~W})$. An outline of the arena is shown in Figure 1. The walls separating the chambers had centered openings (dashed lines in Figure. 1) through which the mice could freely move between chambers. The two side chambers each contained a small cage $(21 \mathrm{~cm} \mathrm{~L} \times$ $10 \mathrm{~cm} \mathrm{~W} \times 22 \mathrm{~cm} \mathrm{H}$ ) built from $1.5 \mathrm{~cm}^{2}$ grid cage wire-mesh. The mouse's movement path was continuously tracked with an automatic video tracking system (BIObserve GmbH, Bonn, Germany). The digitized track coordinates as well as various derived variables such as speed, duration, activity and freezing, were calculated and stored on hard disk for statistical comparison. Movements of the stranger mice in the small cages were not tracked.

The social behavioral test consisted of two consecutive 10-minute trials. Each trial started with the test mouse being placed in the center chamber. For consistency, test mice were set down in the center chamber facing the same general direction (the long outer wall of the arena with the two small cages). During the first trial the two small cages were empty. The test mice explored the arena freely and their exploratory paths were tracked and digitized. Mice were then briefly removed from the arena and placed in a neutral "waiting" cage with fresh bedding while a stranger mouse was placed in one of the two small cages. Cage choice was varied randomly between trials. Then, the test mice were again placed into the center chamber of the arena. Social interactions with the stranger mouse were evaluated based on the amount of time the test mice spent directly in front of the cage with the stranger (see below for details of trajectory analysis).

After completion of each test, the bedding was removed, the arena was wiped down with water and new bedding was put in place. The small metal cages were hosed cleaned with hot water using high pressure and dried before placing them back into the arena. The experimenter was present and observed the mouse's movements for the entire duration of the experiments. The test could not be run unsupervised because occasionally the test mice would attempt to climb onto the cages in the side chambers. If they reached the top of the cage the experimenter used a plastic rod to gently direct them back towards the arena.

\section{Quantitative analysis of exploratory path}

In all tests, mouse movements were analyzed using the automatic video tracking and analysis software Viewer II (BIObserve GmbH, Bonn, Germany). A number of quantitative measures (listed below) were derived from the movement path analysis statistical comparison. These values were calculated for the whole arena (shaded area in Figure 1a) and for each of the two small areas immediately in front of the small cages (shaded rectangular areas in Figure 1b).

Exploratory behavioral variables were defined as follows:

Time spent moving: The percentage of time the mouse was in motion during the 10-minute trials. The speed threshold for motion vs. rest was $0.1 \mathrm{~cm} / \mathrm{sec}$

Speed: Average movement speed over the entire 10-minute trial duration (in $\mathrm{cm} / \mathrm{sec}$ ).

Track length: Total length of the exploratory path (in $\mathrm{cm}$ ).

Area covered: For this analysis the arena was subdivided into small $1.2 \mathrm{~cm} 2$ grids. The total area covered by the exploratory path was calculated as the percentage of grid elements crossed during exploration.

Duration: The time spent at any given site along the path (in sec).

Freezing: The number of events where the animal would be immobile for more than $2 \mathrm{sec}$ (speed $<0.5$ $\mathrm{cm} / \mathrm{s})$.

The following analyses were specific for the areas in from of the small cages:

Number of visits: The number of times the mouse walked into the area in front of the empty or the "stranger's" cage.

Duration: The total amount of time the mice spent inside the areas in front of the empty or the "stranger's" cage.

The results were statistically evaluated using analysis of variance (ANOVA) followed by Tukey Post Hoc test (SPSS v. 17, SPSS Inc., Chicago, IL). F statistics results are reported with between- and within- groups degrees of freedom. All $p$ values are reported as inequalities (e.g. $p \leq 0.01$ ). We used a $95 \%$ confidence interval for significance (i.e. $p<0.05)$. We report Tukey Post Hoc (TPH) test results for all significant pair-wise comparisons 
including the $p$ values and the mean (mdiff) and standard (serr) of the difference to the reference group or test condition.

The exploratory paths as well as speed and duration measurements were visualized in two- and three-dimensional maps (Figure 2). In Figures $2 \mathrm{~A}, \mathrm{~B}, \mathrm{C}$ the exploratory paths are plotted as space vs. time representations. Travel speed and duration are represented in color code in Figures 2D,E,F and 2G,H,I respectively. High speed and duration values are shown in "hot" colors (e.g. red, yellow) and low values in "cold" colors (e.g. blue, green).

\section{Acknowledgements}

This work was supported by grants from the National Institute of Child Health and Human Development (HD057244-02) to DHH and the Angelman Syndrome Foundation to LTR and DHH as well as the National Institute of Neurological Disorders and Stroke (R01NS059902) to LTR. The content of this publication is solely the responsibility of the authors and do not necessarily represent the official views of the $\mathrm{NIH}$.

\section{Author details}

'Department of Anatomy and Neurobiology, University of Tennessee Health Science Center, Memphis, TN 38139, USA. ²Department of Neurology, University of Tennessee Health Science Center, Memphis, TN 38139, USA. ${ }^{3}$ Department of Pediatrics, University of Tennessee Health Science Center, Memphis, TN 38139, USA.

\section{Authors' contributions}

MA carried out the behavioral assays, performed the statistical analysis, contributed to the design of the study and the writing of the manuscript. AS participated in the behavioral assays and contributed to the design of the study. LTR provided the mice and contributed to the preparation of the manuscript. DHH conceived of and coordinated the study and wrote the manuscript. All authors read and approved the final manuscript.

Received: 19 August 2010 Accepted: 14 January 2011

Published: 14 January 2011

\section{References}

1. Williams CA, Angelman H, Clayton-Smith J, Driscoll DJ, Hendrickson JE, Knoll JH, Magenis RE, Schinzel A, Wagstaff J, Whidden EM, Zori RT: Angelman syndrome: consensus for diagnostic criteria. Angelman Syndrome Foundation. Am J Med Genet 1995, 56(2):237-238.

2. Williams CA, Zori RT, Hendrickson J, Stalker H, Marum T, Whidden E, Driscoll DJ: Angelman syndrome. Curr Probl Pediatr 1995, 25(7):216-231.

3. Dan B: Angelman Syndrome. London, UK: Mac Keith Press; 2010.

4. Kishino T, Lalande M, Wagstaff J: UBE3A/E6-AP mutations cause Angelman syndrome. Nat Genet 1997, 15(1):70-73.

5. Matsuura T, Sutcliffe JS, Fang P, Galjaard RJ, Jiang YH, Benton CS, Rommens JM, Beaud, et al: De novo truncating mutations in E6-AP ubiquitin-protein ligase gene (UBE3A) in Angelman syndrome. Nat Genet 1997, 15(1):74-77.

6. Williams CA: Neurological aspects of the Angelman syndrome. Brain Dev 2005, 27(2):88-94.

7. Walz NC: Parent report of stereotyped behaviors, social interaction, and developmental disturbances in individuals with Angelman syndrome. J Autism Dev Disord 2007, 37(5):940-947.

8. Peters SU, Beaudet AL, Madduri N, Bacino CA, et al: Autism in Angelman syndrome: implications for autism research. Clin Genet 2004, 66(6):530-536.

9. Horsler K, Oliver C: Environmental influences on the behavioral phenotype of Angelman syndrome. Am J Ment Retard 2006, 111(5):311-321.

10. Peters SU, Goddard-Finegold J, Beaudet AL, Madduri N, Turcich M, Bacino CA, et al: Cognitive and adaptive behavior profiles of children with Angelman syndrome. Am J Med Genet A 2004, 128A(2):110-113.
11. Jiang $Y H$, Armstrong D, Albrecht $U$, Atkins CM, Noebels JL, Eichele $G$, Sweatt JD, Beaud, et al: Mutation of the Angelman ubiquitin ligase in mice causes increased cytoplasmic p53 and deficits of contextual learning and long-term potentiation. Neuron 1998, 21(4):799-811.

12. Miura K, Kishino T, Li E, Webber H, Dikkes P, Holmes GL, Wagstaff J: Neurobehavioral and electroencephalographic abnormalities in Ube3a maternal-deficient mice. Neurobio/Dis 2002, 9(2):149-159.

13. Heck DH, Zhau Y, Roy S, LeDoux MS, Reiter LT: Analysis of Cerebellar Function in Ube3a Deficient Mice Reveals Novel Genotype Specific Behaviors. HumMolGen 2008, 17(14):2181-2189.

14. Moy SS, Nadler JJ, Young NB, Nonneman RJ, Grossman AW, Murphy DL, D'Ercole AJ, Crawley JN, Magnuson TR, Lauder JM: Social Approach in Genetically-Engineered Mouse Lines Relevant to Autism. Genes Brain Behav 2008, 8:129-142.

15. Zori RT, Hendrickson J, Woolven S, Whidden EM, Gray B, Williams CA: Angelman syndrome: clinical profile. J Child Neurol 1992, 7(3):270-280.

16. Williams CA, Beaudet AL, Clayton-Smith J, Knoll JH, Kyllerman M, Laan LA, Magenis RE, Moncla A, Schinzel AA, Summers JA, Wagstaff J, et al: Angelman syndrome 2005: updated consensus for diagnostic criteria. Am J Med Genet A 2006, 140(5):413-418.

17. Sahoo T, Peters SU, Madduri NS, Glaze DG, German JR, Bird LM, BarbieriWelge R, Bichell TJ, Beaudet AL, Bacino CA, et al: Microarray based comparative genomic hybridization testing in deletion bearing patients with Angelman syndrome: genotype-phenotype correlations. J Med Genet 2006, 43(6):512-516.

18. Veltman MW, Craig EE, Bolton PF: Autism spectrum disorders in PraderWilli and Angelman syndromes: a systematic review. Psychiatr Genet 2005, 15(4):243-254.

19. Williams CA, Driscoll DJ, Dagli Al: Clinical and genetic aspects of Angelman syndrome. Genet Med 2010, 12(7):385-95.

20. Moy SS, Nadler JJ, Young NB, Nonneman RJ, Segall SK, Andrade GM, Crawley JN, Magnuson TR: Social approach and repetitive behavior in eleven inbred mouse strains. Behavioural Brain Research 2008, 191(1):118-129.

doi:10.1186/1471-2156-12-7

Cite this article as: Allensworth et al:: Normal social seeking behavior, hypoactivity and reduced exploratory range in a mouse model of Angelman syndrome. BMC Genetics 2011 12:7.

\section{Submit your next manuscript to BioMed Central and take full advantage of:}

- Convenient online submission

- Thorough peer review

- No space constraints or color figure charges

- Immediate publication on acceptance

- Inclusion in PubMed, CAS, Scopus and Google Scholar

- Research which is freely available for redistribution

Submit your manuscript at www.biomedcentral.com/submit
C Biomed Central 